\title{
Letter
}

\section{Finite-element modelling of no-insulation HTS coils using rotated anisotropic resistivity}

\author{
R.C. Mataira ${ }^{1(D)}$, M.D. Ainslie ${ }^{2}$ (D), R.A. Badcock ${ }^{1}$ (D), C.W. \\ Bumby $^{1}$ (D) \\ ${ }^{1}$ Robinson Research Institute, Victoria University of Wellington, 69 Gracefield Road, \\ Lower Hutt 5010, New Zealand \\ ${ }^{2}$ Bulk Superconductivity Group, Engineering Department, Trumpington Street, \\ Cambridge CB2 1PZ, United Kingdom \\ E-mail: ratu.mataira@vuw.ac.nz
}

December 2019

\begin{abstract}
The no-insulation (NI) winding method is an effective technique for winding coils from high- $T_{\mathrm{c}}$ superconductors (HTS). NI coils are electrically and thermally robust due to their ability to radially bypass current away from the fragile superconducting path when necessary. This avoids stored magnetic energy being entirely discharged on local defects in the HTS tape. However, the increased degrees of freedom for the current distribution makes finite-element modelling of these coils a complicated and multi-level problem. Here we present and validate a 2D axially symmetric model of an NI (or partially insulated) coil that captures all the inherent electromagnetic properties of these coils, including axial vs radial current flow and critical current suppression, and also reproduces the well-known charging and discharging characteristics. The model is validated against previously reported discharge measurements, and is shown to produce results consistent with the expected equivalent-circuit behaviour. Only by solving the NI coil problem with both axial and radial fidelity can the interplay of critical current anisotropy and turn-to-turn current be properly accounted for. The reported FE model will now enable coil designers to simulate key complex behaviours observed in NI coils, such as shielding currents, magnetic field inhomogeneity and remnant field effects.
\end{abstract}

Submitted to: Supercond. Sci. Technol.

\section{Introduction}

High- $T_{c}$ superconducting (HTS) coils wound without insulation (NI coils) were first proposed by Hahn [1], and have many useful properties [2 5] and applications [5 13 . Omitting insulation between the turns of the coil provides both current and heat with alternative pathways through the coil's structure. In the case of current flow this is particularly valuable, as localised faults in the HTS winding can be avoided, with 
current instead flowing into and through adjacent turns. This avoids the potentially destructive quench events that commonly threaten insulated (INS) HTS coils [8, 9, 14. From a macroscopic viewpoint, the extra current paths can be considered as a resistance in parallel with the inductance of the coil. This parallel resistance corresponds to the current flow through one turn of the coil to the next, bypassing the inductive spiral path. However, the introduction of these extra degrees of freedom to the current flow, poses challenges when attempting to model and understand the current and field distribution within an NI coil.

Experiments and modelling $15-23]$ have shown that current flow, and the associated electric and magnetic fields are more complicated than that captured by a simple equivalent-circuit model. However, a full resolution model of such NI coils is practically difficult to implement due to the high aspect ratio of the superconductor and the need to consider the currents flowing normal to the conductor's surface. While progress has been made using circuit grid models (CGM) [18,24 27], these models lose axial fidelity and cannot simulate the associated shielding currents, and associated stress [28], within the HTS windings 23, 29, 31.

Homogenisation 32 35 of conductors is a technique often used to simplify finite element (FE) models of INS coils. Here we combine the idea of conductor homogenisation with the near axial symmetry of the coils to create a 2D axially symmetric model of an NI coil. This model accounts for the spiral structure of the coil, whilst also enabling radial turn-to-turn currents to flow. Although the approach used here can also be applied in 3D, we take full advantage of the symmetry of a circular pancake coil to further reduce the problem to $2 \mathrm{D}$.

\section{Model derivation}

Homogenisation aggregates detailed microscopic properties of the conductor and coil architecture into bulk properties that can be used to represent the homogenised system at larger length scales. This elucidates the essential physics of a system while ignoring extraneous detail 32 35], and hence reduces computational cost. Here we develop a method to represent the spiral geometry of a pancake coil, an inherently axially asymmetric feature, into a bulk property that can be treated with axial symmetry. In INS coils this is normally done by approximating each turn of the coil to be concentric and azimuthal (see figure 1(a) right). This is possible because the spiral current is always equal to the operational current $I_{\mathrm{T}}=I_{\mathrm{op}}$. However this constraint does not hold for an NI coil.

Rather than assume each turn of the coil is azimuthal, we simply homogenise the properties of each portion of a turn across its thickness. This process can be done in coordinates local to each part of the HTS winding. Shown in figure 1, the coordinates are defined by: the surface normal of the tape $\hat{n}$, the tangent to the spiral of the tape $\hat{T}$, and the axial direction $\hat{z}$. In this coordinate system the anisotropic resistivity of the 

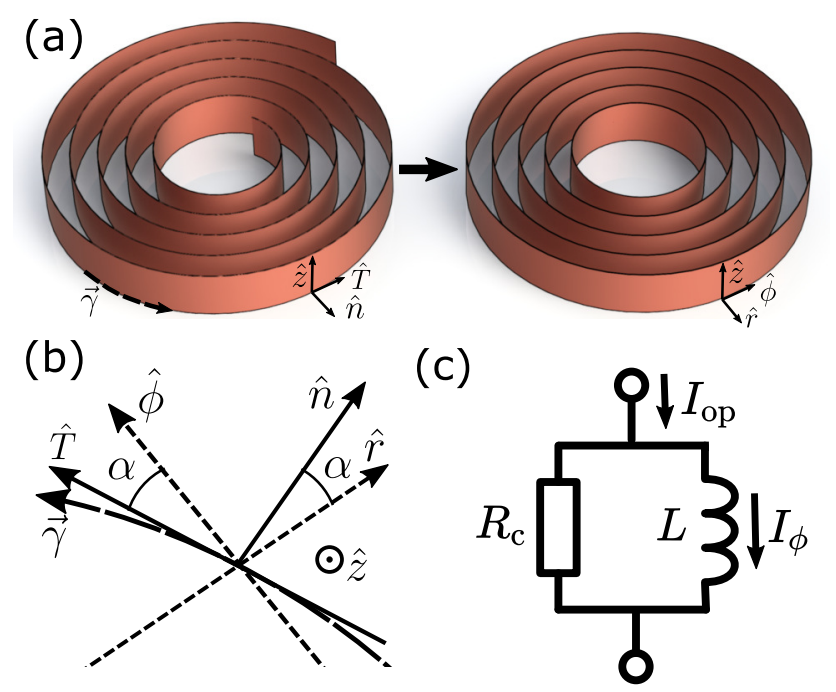

Figure 1. (a) Illustration of the spiral winding of an HTS pancake coil, exaggerating the pitch and spacing of the turns (left), as well as the traditional concentric approximation used to enforce axial symmetry in INS coils (right). (b) A comparison of the locally defined normal-tangential unit vectors, $\hat{n}$ and $\hat{T}$, for the spiral winding vs the cylindrical unit vectors $\hat{r}$ and $\hat{\phi}$, which differ by a small angle $\alpha$. (c) lumped circuit model of the NI coil.

tape is easily understood in the tensor form:

$$
\vec{E}^{\prime}=\boldsymbol{\rho}^{\prime} \vec{J}^{\prime}=\left[\begin{array}{ccc}
\rho_{\mathrm{n}} & 0 & 0 \\
0 & \rho_{\mathrm{sc}} & 0 \\
0 & 0 & \rho_{\mathrm{z}}
\end{array}\right]_{\hat{n}, \hat{T}, \hat{z}} \overrightarrow{J^{\prime}}
$$

where $\vec{E}^{\prime}$ is the electric field, $\vec{J}^{\prime}$ is the current density, and $\boldsymbol{\rho}^{\prime}$ is the resistivity tensor containing: the turn-to-turn resistivity $\rho_{\mathrm{n}}$, the resistivity of the superconductor $\rho_{\mathrm{sc}}$, and the axial resistivity $\rho_{\mathrm{z}}$. Note that' denotes the local coordinate system which is always oriented tangential to the conductor.

At any given point in the global cylindrical coordinate system, $r, \phi, z$, the local tangential coordinates are simply small rotations from the cylindrical system, given by the rotation matrix:

$$
g=\left[\begin{array}{ccc}
\cos (\alpha) & -\sin (\alpha) & 0 \\
\sin (\alpha) & \cos (\alpha) & 0 \\
0 & & 1
\end{array}\right],
$$

where $\alpha$ is the small angle of deviation between the orientation of the spiral winding and the azimuthal direction. This is given geometrically by:

$$
\alpha= \pm \sin ^{-1}\left(\frac{d}{2 \pi r}\right) \sim \pm \frac{d}{2 \pi r} \quad \text { as } \quad \frac{\mathrm{d}}{\mathrm{r}} \rightarrow 0,
$$

where $d$ is the thickness of the turn and the \pm accounts for the winding direction of the coil. 
We can rotate the resistivity tensor from the tangential system, where it is easily understood, into the global cylindrical coordinate system where they can be expressed in an axially symmetric finite element model:

$$
\vec{E}=g \vec{E}^{\prime}=g \boldsymbol{\rho}^{\prime} g^{-1} \vec{J}
$$

where $\vec{E}$ and $\vec{J}$ are the electric field and current density in the global coordinates. Substituting equations (1) and (2) into (4), we then obtain the axially-symmetric resistivity tensor in the global coordinates as:

$$
\boldsymbol{\rho}_{\text {coil }}=g \boldsymbol{\rho}^{\prime} g^{-1}=\left[\begin{array}{ccc}
\rho_{\mathrm{rr}} & \rho_{\mathrm{r} \phi} & 0 \\
\rho_{\phi \mathrm{r}} & \rho_{\phi \phi} & 0 \\
0 & 0 & \rho_{\mathrm{z}}
\end{array}\right]_{\hat{r}, \hat{\phi}, \hat{z}},
$$

where the components are:

$$
\begin{aligned}
& \rho_{\mathrm{rr}}=\rho_{\mathrm{n}} \cos ^{2}(\alpha)+\rho_{\mathrm{sc}} \sin ^{2}(\alpha), \\
& \rho_{\mathrm{r} \phi}=\rho_{\phi \mathrm{r}}=\frac{1}{2}\left(-\rho_{\mathrm{n}}+\rho_{\mathrm{sc}}\right) \sin (2 \alpha), \\
& \rho_{\phi \phi}=\rho_{\mathrm{sc}} \cos ^{2}(\alpha)+\rho_{\mathrm{n}} \sin ^{2}(\alpha) .
\end{aligned}
$$

As $\boldsymbol{\rho}_{\text {coil }}$ is now expressed in the cylindrical coordinate system, we can explicitly associate a resistivity with every point of our model. The off-diagonal terms, $\rho_{\mathrm{r} \phi}$ and $\rho_{\phi \mathrm{r}}$, describe the desired coupling between the radial and azimuthal currents. In principle, with appropriate choices of $\rho_{\mathrm{n}}, \rho_{\mathrm{sc}}$, and $\rho_{\mathrm{z}}$, the full range of coil behaviour can be successfully modelled, from no-insulation $\left(\rho_{\mathrm{n}} \rightarrow 0\right)$, to fully insulated $\left(\rho_{\mathrm{n}} \rightarrow \infty\right)$.

We use the $E-J$ power law [36, 37] to express the nonlinear resistivity of the superconductor:

$$
\rho_{\mathrm{sc}}=\frac{E_{0}}{J_{\mathrm{c}}(B, \theta)^{n}}\left|\overrightarrow{J_{\|}^{\prime}}\right|^{n-1}
$$

where $E_{0}$ is threshold field used to define $J_{c}$ (conventionally $E_{0}=1 \mu \mathrm{V} / \mathrm{cm}$ ), $n$ is the flux creep exponent, $\overrightarrow{J_{\|}^{\prime}}$ is the in plane current density of the HTS tape. $J_{c}(B, \theta)$ is the homogenised engineering critical current density of the tape, expressed as a function of the local magnetic field magnitude $B$ and field angle $\theta$ to the tape. $J_{\mathrm{c}}(B, \theta)$ is given by:

$$
J_{\mathrm{c}}(B, \theta)=\frac{I_{\mathrm{c}}(B, \theta)}{w d},
$$

where $I_{\mathrm{c}}(B, \theta)$ is the measured [38] critical current performance of the conductor, $w$ is the width of the tape and measurement perturbations due to self field effects are ignored [39]. For simplicity the axial resistivity $\rho_{\mathrm{z}}$ is also assumed to be equal to $\rho_{\mathrm{sc}}$. Finally, for $\rho_{\mathrm{n}}$ we simply assume that the contact resistance is evenly distributed over the contact area. We then allocate the contact resistivity as a bulk value:

$$
\rho_{\mathrm{n}}=\frac{R_{\mathrm{ct}}}{d} \text {. }
$$

Unlike the traditional approach, the total current in the coil is not explicitly constrained, rather the coil is connected to a current source, see figure 2, which effectively applies a constraint only on the net radial current $I_{\mathrm{r}}=2 \pi r \int_{-w / 2}^{-w / 2} J_{\mathrm{r}} d z=I_{\mathrm{op}}$. The operating 


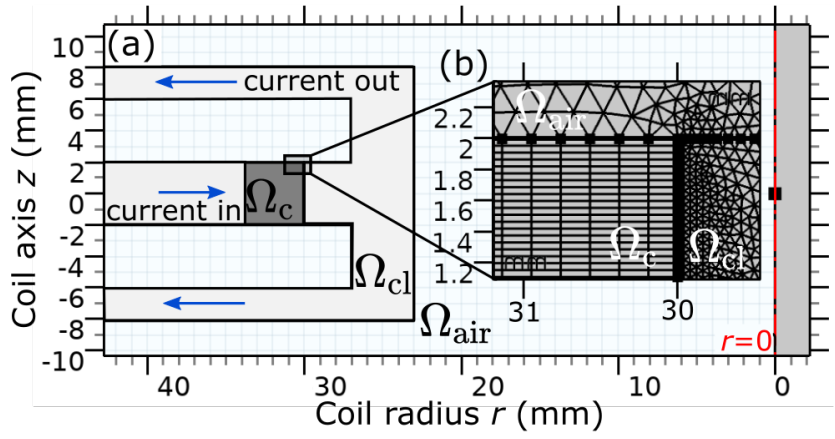

Figure 2. Geometry of the 60 turn coil model. (a) the coil itself is labelled $\Omega_{\mathrm{c}}$ (dark grey), current leads $\Omega_{\mathrm{cl}}$ (light grey) leading to and from the current source (not shown), and air $\Omega_{\mathrm{A}}$ (blue grid). (b) Zoomed view of the coil domain corner showing the rectangular mesh used.

current $I_{\mathrm{op}}$ is transfered to the coil through a normal-conducting bus simulated as copper with a resistivity $\rho_{\mathrm{cu}}=1.67 \times 10^{-8} \Omega \mathrm{m}$.

This model has been implemented in COMSOL multiphysics 5.4 using the $H$ formulation [40,41], chosen for its stability when solving problems with power-law resistivities [42]. However, in principle the approach described above is independent of the numerical system used to solve it.

\section{Model validation}

We validated this FE model against three data sets collected by Wang et al. 43] from a pancake coil with varying number of total turns, $N$. These authors initially performed sudden-discharge measurements on a 60 turn dry-wound pancake coil, before reducing the turn number to make a 40 turn coil on which the measurements were repeated. The process of turn removal was then repeated once more to create a 20 turn coil and on which measurements were also repeated. The reported experiments employed a constant winding tension throughout, in an attempt to maintain a constant contact resistivity $R_{\mathrm{ct}}$ within the coils. Wang et al. analysed their sudden-discharge results using an equivalent circuit model (see figure 1(c)), and their geometric interpretation of the turn-to-turn resistance [43]:

$$
R_{\mathrm{c}}=\sum_{i=1}^{N} R_{i}=\sum_{i=1}^{N} \frac{R_{\mathrm{ct}}}{2 \pi r_{\mathrm{i}} w},
$$

where, $R_{\mathrm{i}}$ is the resistance between the $i^{\text {th }}$ turn and the next.

Table 1 shows the geometric parameters for each of the experimental coils which we have modelled in 2D axial cross-section. Figure 2 shows the model geometry used to deliver current to and from the coil. As the model must conserve axial and radial currents, normal-conducting bus rings are used to connect the inner and outer surfaces of the coil to a simulated current source. This current source is modelled as a highly resistive ring with an imposed current density, which is constrained to flow through the 
Finite-element modelling of no-insulation HTS coils using rotated anisotropic resistivity6

\begin{tabular}{l|ccc} 
Parameter & $\mathrm{N} 20$ & $\mathrm{~N} 40$ & $\mathrm{~N} 60$ \\
\hline Inner radius $R_{\text {in }}(\mathrm{mm})$ & 30.0 & 30.0 & 30.0 \\
Outer radius $R_{\text {out }}(\mathrm{mm})$ & 31.25 & 32.5 & 38.8 \\
Turns $N$ & 20 & 40 & 60 \\
\hline
\end{tabular}

Table 1. Key coil design parameters as reported by Wang et al. The dimensions of the tape used for all coils were width $w=4.0 \mathrm{~mm}$ and thickness $d=63 \mu \mathrm{m}$. [43].

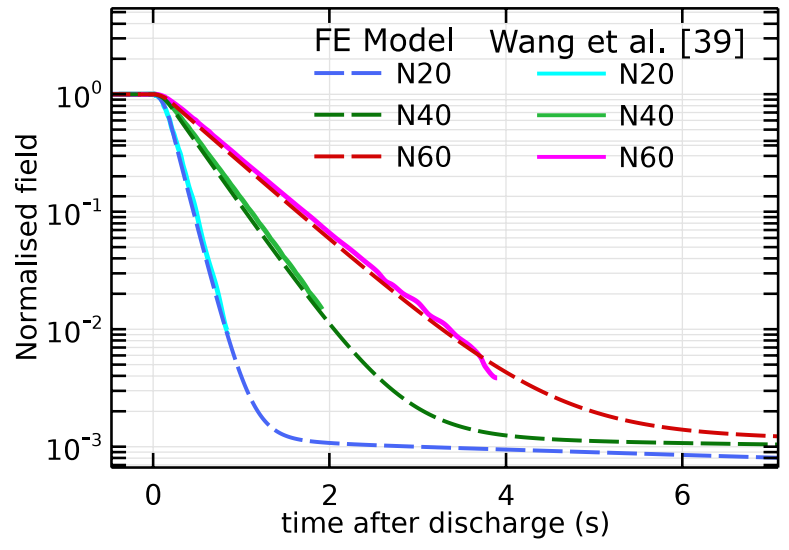

Figure 3. Comparison of experimental decay data ( - ) for all three coils from Wang et al. 43 with the 2D axially symmetric model presented here.

source and hence the connected bus leads. The critical current $I_{\mathrm{c}}(B, \theta)$ for the model is taken from measured data of a SuperPower inc. advanced pinning tape [38, 44], similar to that used by Wang et al.

Experimentally, the azimuthal current cannot be measured directly, so the central field $B_{\mathrm{m}}$, measured by a Hall probe, is reported as an analogue. Figure 3 shows the experimental sudden discharge measurements for each coil, normalised to $B_{0}=\left.B_{\mathrm{m}}\right|_{t=t_{\mathrm{d}}}$, where $t_{\mathrm{d}}$ is the discharge time. The experimental curves (solid) show a slight rounding at the onset of the decay, which may correspond to a finite switching time $\tau_{\mathrm{s}} \approx 200 \mathrm{~ms}$ (although this is not commented on in [43]).

The decay time constant of each coil is determined from the gradient of the straightline sections of each curve shown in figure 3 , using :

$$
\tau=\frac{t_{0}-t_{1}}{\ln \left(\frac{B_{\mathrm{m}}\left(t_{1}\right)}{B_{\mathrm{m}}\left(t_{0}\right)}\right)},
$$

where $t_{0}$ is taken to be $300 \mathrm{~ms}$ (to ensure it is more than than the switching time $\tau_{\mathrm{s}}$ ). To match the data over the whole decay we take $t_{1}=t\left(B_{\mathrm{c}} / B_{0}=2 \%\right)$, to ensure confidence in the fidelity of the data extracted from [43]. Then, the characteristic resistance $R_{\mathrm{c}}$ can be calculated from $\tau=L / R_{\mathrm{c}}$. Hence, values for $\rho_{\mathrm{n}}$ in the model are obtained from (9) and (5a).

The model gives the 3 dashed curves in figure 3, which match the experimental data well, see table 2. This validates the model, and also shows that (for a regime where the broad circuit behaviour is dominant) the FE model delivers macroscopic 
Finite-element modelling of no-insulation HTS coils using rotated anisotropic resistivity7

\begin{tabular}{l|ccc} 
Parameter & $\mathrm{N} 20$ & $\mathrm{~N} 40$ & $\mathrm{~N} 60$ \\
\hline Inductance $L(\mu \mathrm{H})$ & 50.85 & 195.54 & 427.59 \\
$R_{\mathrm{c}}$ (experiment) $(\mu \Omega)$ & 323.8 & 458.7 & 615.8 \\
Time constant $\tau(\mathrm{ms})$ & & & \\
Experiment & 157.1 & 426.3 & 694.4 \\
Model & 153.6 & 419.3 & 683.5 \\
\hline
\end{tabular}

Table 2. Extracted coil parameters, showing comparison of time constants extracted using eq (1) from both the original experimental data in [43], and numerically simulated data produced from the FE model.
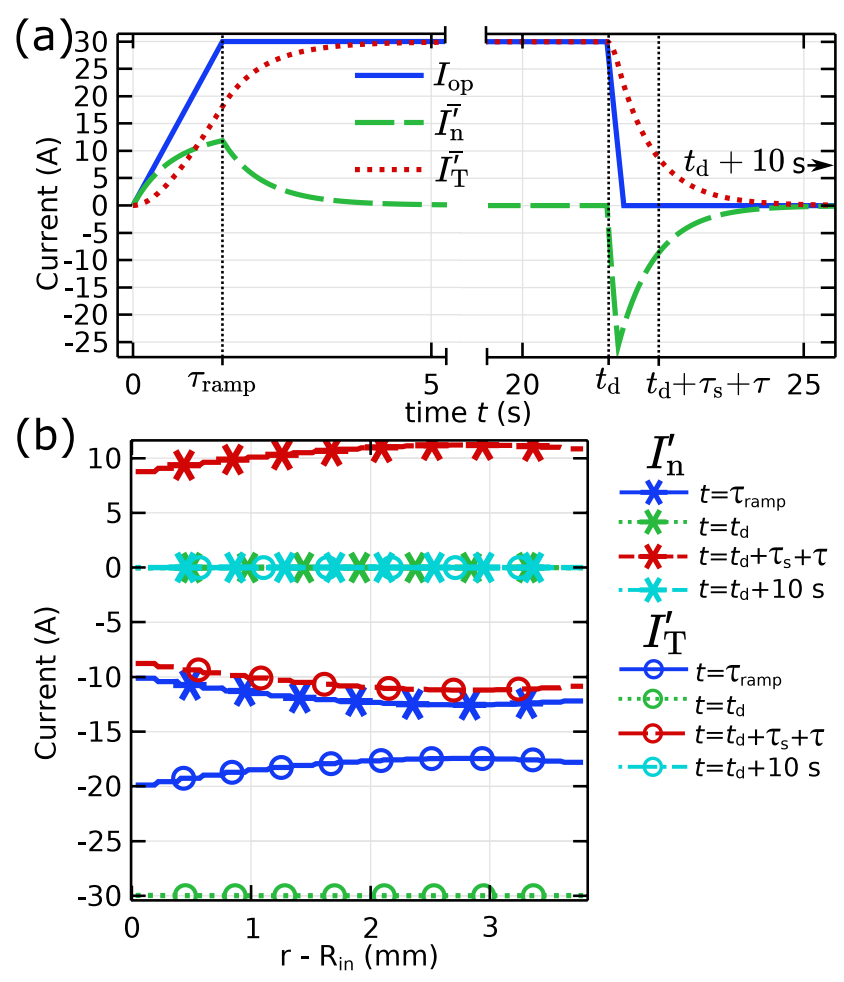

Figure 4. (a) Time evolution in the 60-turn NI coil of the average turn-to-turn current $\bar{I}_{\mathrm{n}}^{\prime}$ (both averaged across the full cross-section of the coil) and the spiral current $\bar{I}_{\mathrm{T}}^{\prime}$. The applied current $I_{\mathrm{op}}$ is also shown. (b) Radial profile across the 60-turn coil of the spiral current in the HTS tape $I_{\mathrm{T}}^{\prime}$ and the turn-to-turn current $I_{\mathrm{n}}^{\prime}$ plotted for each of the four key times highlighted in (a).

values consistent with a simple circuit model [18,43]. However, figure 3 also shows that the model predicts a small remnant magnetisation for each coil. This is simply not captured by any lumped-circuit or circuit-grid models [18], as it requires a model which can describe both azimuthal and axial current distributions in the coil. 


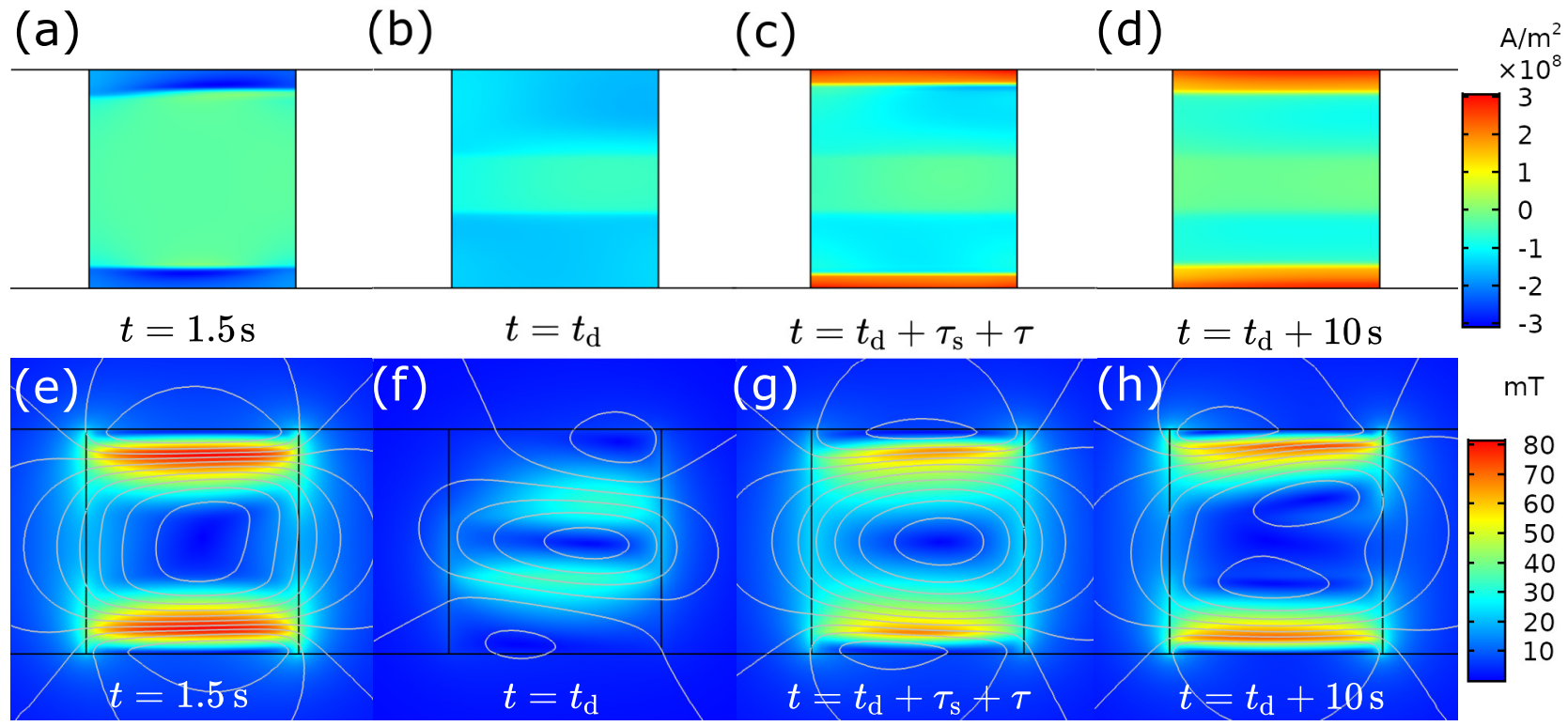

Figure 5. Coil cross-sections showing azimuthal current density $J_{\phi}[(\mathrm{a})-(\mathrm{d})]$ and magnetic field aberration $\Delta B[(\mathrm{e})-(\mathrm{h})]$ at key moments highlighted in figure 4 (a). (a) and (e) show the conclusion of the applied current ramp at $t=1.5 \mathrm{~s}$. (b) and (f) show the moment immediately before the discharge test at $t=t_{\mathrm{d}}, \mathrm{t}$. (c) and (g) show the moment after the switch is fully open, $t=t_{\mathrm{d}}+\tau_{\mathrm{s}}+\tau$, at which point the turnto-turn current achieves its peak value. (d) and (h) show the fully discharged coil at $t=t_{\mathrm{d}}+10 \mathrm{~s}$, where the net currents are zero .

\section{Model interrogation}

Figure 4(a) shows the evolution of the areal-average currents in the 60 turn coil FE model:

$$
\bar{I}_{\mathrm{n}}^{\prime}=\frac{w}{A} \int_{R_{\mathrm{in}}}^{R_{\mathrm{out}}} d r I_{\mathrm{n}}^{\prime}=\frac{2 \pi}{A} \int_{R_{\mathrm{in}}}^{R_{\text {out }}} d r \int_{-w / 2}^{w / 2} d z J_{\mathrm{n}}^{\prime}(z, r)
$$

and:

$$
\overline{I_{\mathrm{T}}^{\prime}}=\frac{w}{A} \int_{R_{\mathrm{in}}}^{R_{\text {out }}} d r I_{\mathrm{T}}^{\prime}=\frac{d}{A} \int_{R_{\mathrm{in}}}^{R_{\text {out }}} d r \int_{-w / 2}^{w / 2} d z J_{\mathrm{T}}^{\prime}(z, r),
$$

where $A$ is the cross sectional area of the coil. As expected, the applied current $I_{\mathrm{op}}$, induces a spiral current $\bar{I}_{\mathrm{T}}^{\prime}$ but with an inductive lag. The overflow turn-to-turn current $\bar{I}_{\mathrm{n}}^{\prime}$ also flows which initially accounts for all of the applied current flow. However this then decreases as a percentage of $I_{\mathrm{op}}$. Once the applied current ramp stops at $t=\tau_{\text {ramp }}$, $\overline{I_{\mathrm{n}}^{\prime}}$ decays exponentially as current adopts the resistance-free spiral pathway. Some time later, $I_{\mathrm{op}}$ is switched off and falls to zero over a switching time $\tau_{\mathrm{s}}$. This causes a rapid increase in $\bar{I}_{\mathrm{n}}^{\prime}$ which is now required to return the radial component of the spiral current. It is noteworthy that $\bar{I}_{\mathrm{n}}$ reaches its peak before the modelled switch is fully open. After this point the average currents then proceed to decay as expected. 
Figure 4(b) shows the spiral current in the HTS tape $I_{\mathrm{T}}^{\prime}$, and the total current flowing between each turn $I_{\mathrm{n}}^{\prime}$, plotted as a function of radial position across the coil at 4 times indicated in figure $4(\mathrm{a})$, see $(11)$ and $(12)$. We see that the normal and spiral currents always sum to a uniform value over the coil, namely $I_{\mathrm{op}}$. However, both $I_{\mathrm{T}}^{\prime}$ and $I_{\mathrm{n}}^{\prime}$ vary with radial position, $r$. This is because both the local resistivity and inductance change across the coil. For example, at $t=t_{\mathrm{d}}+\tau_{\mathrm{s}}+\tau$ both currents are smallest at the inner diameter of the coil, due to the lower $J_{c}(B, \theta)$ and lower mutual inductance at this point. These features are broadly similar to those produced from circuit grid models [18].

Figure 5(a-d) show the azimuthal current densities $J_{\phi}$ for the same moments illustrated in figure 4(a). Figure 5(a) shows that at the conclusion of the applied current ramp, the azimuthal current $J_{\phi}$ flows at the top and bottom edges of the coil, and is approximately zero in the central regions. . Given enough time, $J_{\phi}$ current relaxes into the distribution shown in figure 5(b). Note that $I_{\mathrm{op}}<I_{\mathrm{c}}$ which can be seen as the current has not fully penetrated the coil.

Figure 5(c) shows the distribution of $J_{\phi}$ at a time equal to one time constant after sudden discharge of the coil. Here we see that the total azimuthal current decays through creating new shielding currents of opposite polarity at the top and bottom edge (not by reducing the current density in the interior). This behaviour is reminiscent of the Bean model-like distributions commonly seen in AC loss scenarios [41,45. These azimuthal shielding currents are themselves driven by the tendency of the superconductor to resist changes in magnetic flux. As the opposing azimuthal currents can recirculate in the axial direction, they follow a current path which avoids resistive turn-to-turn transfer. Eventually the total azimuthal current decays to approximately zero whilst leaving the final remnant currents shown in figure $5(d)$.

In cases where field homogeneity is critical, the effect of these recirculating shielding currents must be addressed. Figure 5(e-h) depict the magnetic field aberration for this NI coil, calculated as:

$$
\Delta B=\left|\vec{B}-\overrightarrow{B_{\text {uni }}}\right|
$$

where $\overrightarrow{B_{\text {uni }}}$ is the magnetic field for an identical coil within which the same net azimuthal current $I_{\phi}$ is uniformly distributed throughout. $\Delta B$ thus captures the difference between a typical 'first-cut' magnet design study, (where current is assumed to be uniform through the conductor), and the real situation where non-uniform current distributions occur. Interestingly, we see the largest aberration occurs at the end of the applied current ramp, figure 5(e). Figure 5(f) shows that once the coil is in steady-state operation we still observe non-zero $\Delta B$, due to the incomplete current penetration. During sudden discharge (figure $5(\mathrm{~g}-\mathrm{h})$ ), aberrations are primarily at the top and bottom edges of the coil, and ultimately equals the remnant magnetisation, $\Delta B=B$, in figure 5 (h). For all the results in figure 5 the small asymmetry between the top and bottom of the coil is caused by the anisotropy in $J_{\mathrm{c}}$. 

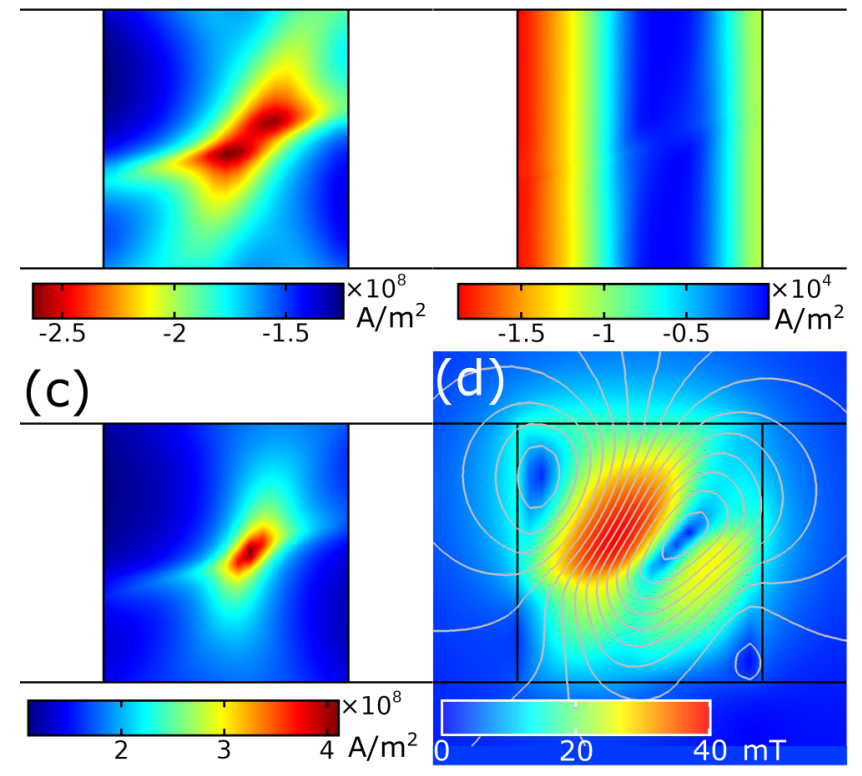

Figure 6. Current densities and field aberration for coil overdrive $I_{\mathrm{op}}=50 \mathrm{~A}>\mathrm{I}_{\mathrm{c}}=$ 43 A. (a) The azimuthal current density. (b) The turn-to-turn bypass current $J_{\mathrm{n}}$. (c) The critical current density $J_{\mathrm{c}}$. (d) The magnetic field aberration $\Delta B$ caused by the nonuniform distribution of current in the coil.

\section{Current overdrive}

Driving NI coils at currents above their critical current $I_{\mathrm{c}, \text { coil }}$ is an interesting way of maximising coil performance [46]. Normally INS HTS coils are limited once some portion of the coil reaches its local $I_{\mathrm{c}}(B, \theta)$. However, in an NI coil the applied current can radially bypass such regions and hence continue to be increased. Our model is uniquely capable of tackling this problem, by solving the current bypass and $J_{\mathrm{c}}(B, \theta)$ behaviours simultaneously.

Figure 6 shows the effect of driving the 60-turn NI coil well past its $I_{\mathrm{c}}$ to $I_{\mathrm{op}}=1.16 \times I_{\mathrm{c}, \mathrm{coil}}[43]$. As shown in figure 6 (a), the distribution of $J_{\phi}$ becomes highly nontrivial. Even at $50 \mathrm{~A}$ above $I_{\mathrm{c}, \text { coil }}$, the coil does not appear to be completely saturated, as evidenced by the absence of radial bypass current in the interior region of the coil, see figure 6(b). This is reinforced by the fact that $J_{\phi}<J_{\mathrm{c}}$ in much of that region of the coil, compare figure 6 (a) and (c). Finally $\Delta B$ is shown in figure 6(d) where we see that the aberration is of a similar magnitude to that observed in the under-driven coil in steady-state operation (figure $5(\mathrm{f})$ ). However, in the over-driven case the spatial distribution of $\Delta B$ is highly asymmetric, and oriented in a different direction. Again we attribute this behaviour to the anisotropy in $J_{\mathrm{c}}$. 
Finite-element modelling of no-insulation HTS coils using rotated anisotropic resistivity11

\section{Conclusion}

The simulation of shielding currents, field inhomogeneities and remnant fields are integral to the development and design of future NI coils. Here, we have derived and demonstrated a computationally and conceptually simple FE approach which captures and predicts the full electromagnetic behaviour of an NI coil. This model correctly replicates experimental 'sudden-discharge' data reported in literature [43], and delivers macroscopic values which correspond to lumped equivalent-circuit models. The model also produces the interchange between the turn-to-turn current and spiral current across the width of the coil, as seen in circuit grid models [18]. However, the power of this FE model lies in its ability to predict the evolution of fields and current at each point within the full coil cross-section. Interrogation of its results reveal the evolution of currents within the cross-section of the NI coil during current ramping, steady-state operation and sudden discharge - with full radial and axial fidelity. Finally, we have also explored a model coil when operated in an 'over-driven' regime, $I_{\mathrm{op}}>I_{\mathrm{c}, \text { coil }}$, revealing the highly nontrivial azimuthal current densities.

This is all achieved within a single, cohesive finite-element model which requires no inter-model coupling. Indeed, no a priori azimuthal current constraints are ever imposed on the coil to produce the delivered results. As a result this approach could be readily incorporated into other coupled physics problems such as thermal and stress simulation. We expect an easy extension to 3D and coupled physics problems.

\section{Acknowledgements}

The authors would like to thank Konstantinos Bouloukakis and Ben Parkinson for useful discussion regarding their own NI coils. M.A. acknowledges financial support from an EPSRC Early Career Fellowship EP/P020313/1. All data are provided in full in sections 3 through 5 of this paper.

\section{References}

[1] Hahn S, Park D K, Bascunan J and Iwasa Y 2011 HTS Pancake Coils Without Turn-to-Turn Insulation IEEE Trans. Appl. Supercond. 21 1592-1595 ISSN 2378-7074

[2] Hahn S, Radcliff K, Kim K, Kim S, Hu X, Kim K, Abraimov D V and Jaroszynski J 2016 'Defect-irrelevant' behavior of a no-insulation pancake coil wound with REBCO tapes containing multiple defects Supercond. Sci. Technol. 29105017 ISSN 0953-2048, 1361-6668

[3] Wang Y, Chan W and Schwartz J 2016 Self-protection mechanisms in no-insulation (RE)Ba2cu3oxhigh temperature superconductor pancake coils Supercond. Sci. Technol. 29 045007 ISSN 0953-2048

[4] Liu D, Zhang W, Yong H and Zhou Y 2018 Thermal stability and mechanical behavior in noinsulation high-temperature superconducting pancake coils Supercond. Sci. Technol. 31085010 ISSN 0953-2048

[5] Hahn S, Kim K, Kim K, Hu X, Painter T, Dixon I, Kim S, Bhattarai K R, Noguchi S, Jaroszynski $\mathrm{J}$ and Larbalestier D C 2019 45.5-tesla direct-current magnetic field generated with a hightemperature superconducting magnet Nature 570 496-499 ISSN 0028-0836, 1476-4687 
Finite-element modelling of no-insulation HTS coils using rotated anisotropic resistivity12

[6] Iwasa Y and Hahn S 2013 First-cut design of an all-superconducting 100-T direct current magnet Appl. Phys. Lett. 103253507 ISSN 0003-6951, 1077-3118

[7] Hahn S, Kim Y, Keun Park D, Kim K, Voccio J P, Bascuñán J and Iwasa Y 2013 No-insulation multi-width winding technique for high temperature superconducting magnet Appl. Phys. Lett. 103173511 ISSN 0003-6951, 1077-3118

[8] Weijers H W, Markiewicz W D, Voran A J, Gundlach S R, Sheppard W R, Jarvis B, Johnson Z L, Noyes P D, Lu J, Kandel H, Bai H, Gavrilin A V, Viouchkov Y L, Larbalestier D C and Abraimov D V 2014 Progress in the Development of a Superconducting 32 T Magnet With REBCO High Field Coils IEEE Trans. Appl. Supercond. 244301805 ISSN 2378-7074

[9] Parkinson B 2016 Design considerations and experimental results for MRI systems using HTS magnets Supercond. Sci. Technol. 30014009 ISSN 0953-2048

[10] Kim K, Bhattarai K R, Jang J Y, Hwang Y J, Kim K, Yoon S, Lee S and Hahn S 2017 Design and performance estimation of a $35 \mathrm{~T} 40 \mathrm{~mm}$ no-insulation all-REBCO user magnet Supercond. Sci. Technol. 30065008 ISSN 0953-2048, 1361-6668

[11] Bong U, An S, Voccio J, Kim J, Lee J T, Lee J, Han K J, Lee H and Hahn S 2019 A Design Study on 40 MW Synchronous Motor With No-Insulation HTS Field Winding IEEE Trans. Appl. Supercond. 295203706 ISSN 2378-7074

[12] Yoon S, Kim J, Cheon K, Lee H, Hahn S and Moon S H 201626 T 35 mm all-GdBa2cu3o7-xmultiwidth no-insulation superconducting magnet Supercond. Sci. Technol. 29 04LT04 ISSN 09532048

[13] Wang D, Zhang Z, Zhang X, Jiang D, Dong C, Huang H, Chen W, Xu Q and Ma Y 2019 First performance test of a $30 \mathrm{~mm}$ iron-based superconductor single pancake coil under a $24 \mathrm{~T}$ background field Supercond. Sci. Technol. 32 04LT01 ISSN 0953-2048, 1361-6668

[14] Ma D X, Matsumoto S, Teranishi R, Ohmura T, Kiyoshi T, Otsuka A, Hamada M, Maeda H, Yanagisawa Y, Nakagome H and Suematsu H 2014 Degradation analysis of REBCO coils Supercond. Sci. Technol. 27085014 ISSN 0953-2048, 1361-6668

[15] Choi S, Jo H C, Hwang Y J, Hahn S and Ko T K 2012 A Study on the No Insulation Winding Method of the HTS Coil IEEE Trans. Appl. Supercond. 224904004 ISSN 1051-8223, 1558-2515, 2378-7074

[16] Kim K, Hahn S, Kim Y, Yang D G, Song J B, Bascuñán J, Lee H and Iwasa Y 2014 Effect of Winding Tension on Electrical Behaviors of a No-Insulation ReBCO Pancake Coil IEEE Trans. Appl. Supercond. 244600605 ISSN 1051-8223, 1558-2515, 2378-7074

[17] Noguchi S, Hahn S, Ueda H, Kim S and Ishiyama A 2018 An Extended Thin Approximation Method to Simulate Screening Current Induced in REBCO Coils IEEE Trans. Magn. 547201904 ISSN 0018-9464, 1941-0069

[18] Wang Y, Song H, Xu D, Li Z Y, Jin Z and Hong Z 2015 An equivalent circuit grid model for no-insulation HTS pancake coils Supercond. Sci. Technol. 28045017 ISSN 0953-2048, 1361-6668

[19] Bhattarai K R, Kim K, Kim K, Radcliff K, Hu X, Im C, Painter T, Dixon I, Larbalestier D, Lee S and Hahn S 2020 Understanding quench in no-insulation (NI) REBCO magnets through experiments and simulations Superconductor Science and Technology 33035002

[20] Noguchi S, Monma K, Iwai S, Miyazaki H, Tosaka T, Nomura S, Kurusu T, Ueda H, Ishiyama A, Urayama S and Fukuyama H 2016 Experiment and Simulation of Impregnated No-Insulation REBCO Pancake Coil IEEE Trans. Appl. Supercond. 264601305 ISSN 2378-7074

[21] Lécrevisse T, Badel A, Benkel T, Chaud X, Fazilleau P and Tixador P 2018 Metal-as-insulation variant of no-insulation HTS winding technique: pancake tests under high background magnetic field and high current at 4.2 K Supercond. Sci. Technol. 31055008 ISSN 0953-2048, 1361-6668

[22] Sung Lee T, Jin Hwang Y, Lee J, Seung Lee W, Kim J, Hyun Song S, Cheol Ahn M and Kuk Ko T 2014 The effects of co-wound Kapton, stainless steel and copper, in comparison with no insulation, on the time constant and stability of GdBCO pancake coils Supercond. Sci. Technol. 27 065018 ISSN 0953-2048, 1361-6668

[23] Wang L, Wang Q, Liu J, Wang H, Hu X and Chen P 2017 Screening Current-Induced Magnetic 
Finite-element modelling of no-insulation HTS coils using rotated anisotropic resistivity13

Field in a Noninsulated GdBCO HTS Coil for a 24 T All-Superconducting Magnet IEEE Trans. Appl. Supercond. 278200106 ISSN 2378-7074

[24] Wang T, Noguchi S, Wang X, Arakawa I, Minami K, Monma K, Ishiyama A, Hahn S and Iwasa Y 2015 Analyses of Transient Behaviors of No-Insulation REBCO Pancake Coils During Sudden Discharging and Overcurrent IEEE Trans. Appl. Supercond. 254603409 ISSN 2378-7074

[25] Wang X, Wang T, Nakada E, Ishiyama A, Itoh R and Noguchi S 2015 Charging Behavior in NoInsulation REBCO Pancake Coils IEEE Trans. Appl. Supercond. 254601805 ISSN 2378-7074

[26] Noguchi S and Hahn S 2019 Torque Simulation on NI REBCO Pancake Coils during Quench $J$. Phys.: Conf. Ser. 1293012061 ISSN 1742-6596

[27] Zhang Y, Tang Y, Xia Z, Ren L and Li J 2018 Investigation on Performance of No-Insulation Coil Considering the Influence of Stress Distribution on Radial Characteristic Resistivity IEEE Trans. Appl. Supercond. 284604007 ISSN 2378-7074

[28] Li Y, Park D, Yan Y, Choi Y, Lee J, Michael P C, Chen S, Qu T, Bascuñán J and Iwasa Y 2019 Magnetization and screening current in an $800 \mathrm{MHz}$ (18.8 t) REBCO nuclear magnetic resonance insert magnet: experimental results and numerical analysis Supercond. Sci. Technol. 32105007

[29] Yang D G, Kim K L, Choi Y H, Kwon O J, Park Y J and Lee H G 2013 Screening current-induced field in non-insulated GdBCO pancake coil Supercond. Sci. Technol. 26 105025 ISSN 0953-2048

[30] Kim S, Lee C, Bang J and Hahn S 2018 Manipulation of screening currents in an (RE)Ba2cu3o7-x superconducting magnet Mater. Res. Express 6026004 ISSN 2053-1591

[31] Noguchi S, Ueda H, Hahn S, Ishiyama A and Iwasa Y 2019 A simple screening current-induced magnetic field estimation method for REBCO pancake coils Supercond. Sci. Technol. 32045007 ISSN 0953-2048, 1361-6668

[32] Clem J R, Claassen J H and Mawatari Y 2007 AC losses in a finiteZstack using an anisotropic homogeneous-medium approximation Supercond. Sci. and Technol. 20 1130-1139

[33] Prigozhin L and Sokolovsky V 2011 Computing AC losses in stacks of high-temperature superconducting tapes Supercond. Sci. Technol. 24075012

[34] Zermeno V M R, Abrahamsen A B, Mijatovic N, Jensen B B and Sørensen M P 2013 Calculation of alternating current losses in stacks and coils made of second generation high temperature superconducting tapes for large scale applications J. Appl. Phys. 114173901 ISSN 0021-8979, 1089-7550

[35] Pardo E 2013 Calculation of AC loss in coated conductor coils with a large number of turns Supercond. Sci. Technol. 26105017

[36] Plummer C and Evetts J 1987 Dependence of the shape of the resistive transition on composite inhomogeneity in multifilamentary wires IEEE Trans. Magn. 23 1179-1182 ISSN 1941-0069

[37] Rhyner J 1993 Magnetic properties and AC-losses of superconductors with power law current-voltage characteristics Physica C 212292 - 300 ISSN 0921-4534

[38] Wimbush S C and Strickland N M 2017 A public database of high-temperature superconductor critical current data IEEE Trans. Appl. Supercond. 278000105 ISSN 1051-8223

[39] Zermeño V M R, Habelok K, Stepień M and Grilli F 2017 A parameter-free method to extract the superconductor's j c ( b , $\theta$ ) field-dependence from in-field current-voltage characteristics of high temperature superconductor tapes Supercond. Sci. Technol. 30034001

[40] Hong Z, Campbell A M and Coombs T A 2006 Numerical solution of critical state in superconductivity by finite element software Supercond. Sci. Technol. 19 1246-1252

[41] Ainslie M D, Bumby C W, Jiang Z, Toyomoto R and Amemiya N 2018 Numerical modelling of dynamic resistance in high-temperature superconducting coated-conductor wires Superconductor Science and Technology $\mathbf{3 1} 074003$

[42] Dular J, Geuzaine C and Vanderheyden B 2020 Finite-element formulations for systems with high-temperature superconductors IEEE Trans. Appl. Supercond. 308200113 ISSN 2378-7074

[43] Wang X, Hahn S, Kim Y, Bascuñán J, Voccio J, Lee H and Iwasa Y 2013 Turn-to-turn contact characteristics for an equivalent circuit model of no-insulation ReBCO pancake coil Supercond. 
Sci. Technol. 26035012 ISSN 0953-2048, 1361-6668

[44] Brooks J, Ainslie M, Jiang Z, Pantoja A, Badcock R and Bumby C The transient voltage response of rebco coated conductors exhibiting dynamic resistance accepted to Supercond. Sci. Tech.

[45] Andrianov V V, Zenkevich V B, Kurguzov V V, Sychev V V and Ternovskii F F 1970 Effective Resistance of an Imperfect Type II Superconductor in an Oscillating Magnetic Field Sov. J. Exp. Theor. Phys. 31815

[46] Brittles G, Slade R and Bateman R Testing of Quality Assessment (QA) HTS Magnets at Tokamak Energy Proceedings of MT26 Indico:Tue-Af-Po2.14-12 [11] URL https://indico.cern.ch/ event/763185/contributions/3415944/ 